\title{
EYNARD-MEHTA THEOREM, SCHUR PROCESS, AND THEIR PFAFFIAN ANALOGS
}

\author{
Alexei Borodin and Eric M. Rains
}

February 11, 2005

\begin{abstract}
We give simple linear algebraic proofs of Eynard-Mehta theorem, Okounkov-Reshetikhin formula for the correlation kernel of the Schur process, and Pfaffian analogs of these results. We also discuss certain general properties of the spaces of all determinantal and Pfaffian processes on a given finite set.
\end{abstract}

\section{INTRODUCTION}

The goal of this note is to give simple proofs of Eynard-Mehta theorem, Okounkov-Reshetikhin formula for the correlation kernel of the Schur process, and Pfaffian analogs of these results.

The Eynard-Mehta theorem [EM] provides a determinantal formula for marginal distributions of probability measures on $n k$-point configurations

$$
\left\{x_{1}^{(1)}, \ldots, x_{n}^{(1)}\right\} \cup \cdots \cup\left\{x_{1}^{(k)}, \ldots, x_{n}^{(k)}\right\}
$$

of the form

$$
\text { const } \cdot \operatorname{det} \phi_{i}\left(x_{j}^{(1)}\right) \operatorname{det} W_{1}\left(x_{i}^{(1)}, x_{j}^{(2)}\right) \cdots \operatorname{det} W_{k-1}\left(x_{i}^{(k-1)}, x_{j}^{(k)}\right) \operatorname{det} \psi_{i}\left(x_{j}^{(k)}\right) .
$$

The formula was initially derived for computing the spectral correlations of coupled random matrices, but has been used for a number of other purposes since then. Alternative proofs of the formula can be found in [NF1], [TW2], [J2].

The Pfaffian analog of this result gives a Pfaffian formula for marginal distributions of probability measures of the form

$$
\text { const. } \operatorname{Pf} \epsilon\left(x_{i}^{(1)}, x_{j}^{(1)}\right) \operatorname{det} V_{1}\left(x_{i}^{(1)}, x_{j}^{(2)}\right) \cdots \operatorname{det} V_{k-1}\left(x_{i}^{(k-1)}, x_{j}^{(k)}\right) \operatorname{det} \xi_{i}\left(x_{j}^{(k)}\right) .
$$

A variant of this formula relevant for evaluating the dynamical correlation functions of the orthogonal-unitary and symplectic-unitary random matrix transitions, was proved in [FNH], [NF2].

The Schur process was introduced by Okounkov-Reshetikhin in [OR]. It is a probability measure on (generally speaking, infinite) sequences of partitions, which in the case of finite sequences

$$
\varnothing \subset \lambda^{(1)} \supset \mu^{(1)} \subset \lambda^{(2)} \supset \mu^{(2)} \subset \cdots \supset \mu^{(T-1)} \subset \lambda^{(T)} \supset \varnothing
$$


takes the form

$$
\text { const } \cdot s_{\lambda^{(1)}}\left(\rho_{0}^{+}\right) s_{\lambda^{(1)} / \mu^{(1)}}\left(\rho_{1}^{-}\right) s_{\lambda^{(2)} / \mu^{(1)}}\left(\rho_{1}^{+}\right) \cdots s_{\lambda^{(T)} / \mu^{(T-1)}}\left(\rho_{T-1}^{+}\right) s_{\lambda^{(T)}}\left(\rho_{T}^{-}\right)
$$

Here $s_{\lambda}, s_{\lambda / \mu}$ are the usual and skew Schur functions, and $\rho_{i}^{ \pm}$are specializations of the algebra of symmetric functions. Thanks to (Jacobi-Trudi) determinantal formulas for $s_{\lambda}, s_{\lambda / \mu}$, the Eynard-Mehta theorem can be applied to evaluating the correlation functions of the Schur process. One way of doing that is explained in [J2], although the original derivation of the correlation functions in [OR] uses different methods. We give another way of deriving the Okounkov-Reshetikhin formula for the correlation kernel of the Schur process from the Eynard-Mehta theorem.

The Schur process has been used for analyzing uniformly distributed plane partitions (or 3d Young diagrams) [OR], polynuclear growth processes [J2], and domino tilings of the Aztec diamond [J3].

Quite similarly, using the Pfaffian analog of the Eynard-Mehta result, we obtain the Pfaffian structure and a formula for the correlation kernel for the Pfaffian Schur process, which associates to the sequence of partitions above the weight

$$
\text { const } \cdot \tau_{\lambda^{(1)}}\left(\rho_{0}^{+}\right) s_{\lambda^{(1)} / \mu^{(1)}}\left(\rho_{1}^{-}\right) s_{\lambda^{(2)} / \mu^{(1)}}\left(\rho_{1}^{+}\right) \cdots s_{\lambda^{(n)} / \mu^{(n-1)}}\left(\rho_{n-1}^{+}\right) s_{\lambda^{(n)}}\left(\rho_{n}^{-}\right) .
$$

where the symmetric functions $\tau_{\lambda}$ are defined by $\tau_{\lambda}=\sum_{\kappa^{\prime} \text { even }} s_{\lambda / \kappa}$. These functions have a Pfaffian representation, see Lemma 3.1 below, which plays a key role in the proof.

The Pfaffian Schur process was essentially introduced by Sasamoto-Imamura [SI], with $\rho_{0}^{+}$specializing the symmetric functions into one variable equal to 1 . They computed the correlation functions and used them for asymptotic analysis of polynuclear growth processes with a wall. The Pfaffian Schur process can also be used for studying tiling models with a symmetry condition, but further explanations of this connection go beyond the goals of this paper.

Following our treatment of the Pfaffian Schur process, Matsumoto in [Mat2] gave a linear algebraic proof of his formulas for the correlation functions of the shifted Schur measure, see [Mat1] for the initial derivation. The shifted Schur measures were first introduced in [TW3].

The basic tool of our proofs is the computation of inverse of the "Gram matrix" of inner products for the corresponding model. Similar ideas have been previously used in [TW1], [B], [R], [J1], [J2], [J4].

In the last section of this paper we also discuss certain general properties of the spaces of all determinantal and Pfaffian processes on a given finite set.

This research was partially conducted during the period one of the authors (A.B.) served as a Clay Mathematics Institute Research Fellow. He was also partially supported by the NSF grant DMS-0402047.

\section{Eynard-Mehta theorem and its Pfaffian analog}

Let $\mathfrak{X}$ be a finite set. A random point process on $\mathfrak{X}$ is a probability measure on the set $2^{\mathfrak{X}}$ of all subsets of $\mathfrak{X}$. The subsets of $\mathfrak{X}$ will also be called point configurations. Let $L$ be a $|\mathfrak{X}| \times|\mathfrak{X}|$ matrix whose rows and column are parameterized by points of $\mathfrak{X}$. For any subset $X \subset \mathfrak{X}$ we will denote by $L_{X}$ the symmetric submatrix of $L$ corresponding to $X$ :

$$
L_{X}=\left\|L\left(x_{i}, x_{j}\right)\right\|_{x_{i}, x_{j} \in X}
$$


If determinants of all such submatrices are nonnegative (e.g., if $L$ is positive definite), one can define a random point process on $\mathfrak{X}$ by

$$
\operatorname{Prob}\{X\}=\frac{\operatorname{det} L_{X}}{\operatorname{det}(\mathbf{1}+L)}, \quad X \subset \mathfrak{X} .
$$

This process is called the $L$-ensemble.

A random point process is called determinantal if there exists a $|\mathfrak{X}| \times|\mathfrak{X}|$ matrix $K$ with rows and columns parameterized by points of $\mathfrak{X}$ such that the correlation functions

$$
\rho(Y)=\operatorname{Prob}\left\{X \in 2^{\mathfrak{X}} \mid Y \subset X\right\}, \quad Y \subset \mathfrak{X},
$$

of the process have determinantal form: $\rho(Y)=\operatorname{det} K_{Y}$. The matrix $K$ is often called the correlation kernel of the process. ${ }^{1}$

Proposition 1.1 [Ma, DVJ]. The L-ensemble as defined above is a determinantal point process with the correlation kernel $K$ given by $K=L(\mathbf{1}+L)^{-1}$.

Take a nonempty subset $\mathfrak{Y}$ of $\mathfrak{X}$ and, given an $L$-ensemble on $\mathfrak{X}$, define a new random point process on $\mathfrak{Y}$ by considering the intersections of the random point configurations $X \subset \mathfrak{X}$ of the $L$-ensemble with $\mathfrak{Y}$, provided that these point configurations contain the complement $\overline{\mathfrak{Y}}$ of $\mathfrak{Y}$ in $\mathfrak{X}$. It is not hard to see that this new process can be defined by

$$
\operatorname{Prob}\{Y\}=\frac{\operatorname{det} L_{Y \cup \bar{Y}}}{\operatorname{det}\left(\mathbf{1}_{\mathfrak{Y}}+L\right)}, \quad Y \subset \mathfrak{Y} .
$$

Here $\mathbf{1}_{\mathfrak{Y}}$ is the block matrix $\left[\begin{array}{ll}\mathbf{1} & 0 \\ 0 & 0\end{array}\right]$ where the blocks correspond to the splitting $\mathfrak{X}=\mathfrak{Y} \sqcup \overline{\mathfrak{Y}}$. We call this new process the conditional L-ensemble.

Proposition 1.2. The conditional L-ensemble is a determinantal point process with the correlation kernel given by

$$
K=\mathbf{1}_{\mathfrak{Y}}-\left.\left(\mathbf{1}_{\mathfrak{Y}}+L\right)^{-1}\right|_{\mathfrak{Y} \times \mathfrak{Y}} .
$$

Note that for $\mathfrak{Y}=\mathfrak{X}$ this statement coincides with Proposition 1.1.

Proof. Using the fact that if $B=A^{-1}$ then $\operatorname{det} B_{X}=\frac{\operatorname{det} A_{\bar{X}}}{\operatorname{det} A}$, for any $Y \in 2^{\mathfrak{Y}}$ we obtain

$$
\begin{aligned}
& \operatorname{det} K_{Y}=\sum_{X \subset Y}(-1)^{|X|} \operatorname{det}\left(\left(\mathbf{1}_{\mathfrak{Y}}+L\right)^{-1}\right)_{X}=\sum_{Z=\bar{X} \supset \bar{Y}}(-1)^{|X|} \frac{\operatorname{det}\left(\mathbf{1}_{\mathfrak{Y}}+L\right)_{Z}}{\operatorname{det}\left(\mathbf{1}_{\mathfrak{Y}}+L\right)} \\
= & \sum_{Z=\bar{X} \supset \bar{Y}}(-1)^{|X|} \operatorname{Prob}\{\text { all points of the random point configuration are in } Z\} \\
= & \sum_{X \subset Y}(-1)^{|X|} \operatorname{Prob}\{X \text { has no points of the random point configuration }\}=\rho(Y)
\end{aligned}
$$

\footnotetext{
${ }^{1}$ Note that the correlation kernel is not defined uniquely; conjugation of $K$ by a diagonal matrix does not change the minors $\operatorname{det} K_{Y}$.
} 
where the last equality is the inclusion-exclusion principle.

Let us now state the Eynard-Mehta theorem [EM]. Other proofs of this theorem are given in [NF1], [J2], [TW2].

Consider a random point process on a disjoint union of $k$ (finite) sets $\mathfrak{X}^{(1)} \cup$ $\cdots \cup \mathfrak{X}^{(k)}$ which lives on $n k$-point configurations with exactly $n$ points in each $\mathfrak{X}^{(i)}, i=1, \ldots, k$, defined by the condition that the probability of any such point configuration equals

$$
\begin{aligned}
\operatorname{Prob}\left\{\left\{x_{i}^{(1)}\right\}_{i=1}^{n} \cup \cdots \cup\left\{x_{i}^{(k)}\right\}_{i=1}^{n}\right\} \\
=\text { const } \cdot \operatorname{det}_{1 \leq i, j \leq n}\left[\phi_{i}\left(x_{j}^{(1)}\right)\right] \cdot \operatorname{det}_{1 \leq i, j \leq n}\left[W_{1}\left(x_{i}^{(1)}, x_{j}^{(2)}\right)\right] \cdots \\
\quad \cdots \operatorname{det}_{1 \leq i, j \leq n}\left[W_{k-1}\left(x_{i}^{(k-1)}, x_{j}^{(k)}\right)\right] \operatorname{det}_{1 \leq i, j \leq n}\left[\psi_{i}\left(x_{j}^{(k)}\right)\right] .
\end{aligned}
$$

Here $\left\{\phi_{i}\right\}_{i=1, \ldots, n}$, are some functions on $\mathfrak{X}^{(1)},\left\{\psi_{i}\right\}_{i=1, \ldots, n}$, are some functions on $\mathfrak{X}^{(k)}$, and $\left\{W_{m}\right\}_{m=1, \ldots, k-1}$, are matrices with rows parameterized by points of $\mathfrak{X}^{(m)}$ and columns parameterized by points of $\mathfrak{X}^{(m+1)}$. The normalization constant in the right-hand side of (1.2) is chosen in such a way that the total mass of all admissible point configurations is equal to 1 . We do not address the problem of positivity of (1.2) as it does not play any role in the sequel. It suffices to assume that the normalization constant is finite (the total mass is nonzero).

It is convenient to organize the functions $\phi_{i}$ and $\psi_{i}$ into two matrices $\Phi$ and $\Psi$, the rows of $\Phi$ and the columns of $\Psi$ are parameterized by $\{1, \ldots, n\}$, the columns of $\Phi$ are parameterized by points of $\mathfrak{X}^{(1)}$, and the rows of $\Psi$ are parameterized by points of $\mathfrak{X}^{(k)}$. The corresponding matrix elements are just the values of $\phi_{i}$ and $\psi_{i}$ at the corresponding points.

Lemma 1.3. The sum of the right-hand sides of (1.2) with "const" removed, taken over all possible point configurations is equal to $\operatorname{det} M$, where

$$
M=\Phi W_{1} \cdots W_{k-1} \Psi .
$$

Thus, const in (1.2) is equal to $\operatorname{det} M^{-1}$, provided that $\operatorname{det} M \neq 0$.

Proof. Follows from the well known Cauchy-Binet formula.

In what follows we always assume that $M$ is invertible, that is $\operatorname{det} M \neq 0$.

Set

$$
W_{[i, j)}= \begin{cases}W_{i} \cdots W_{j-1}, & i<j, \\ 0, & i \geq j .\end{cases}
$$

Theorem 1.4 (Eynard-Mehta). The random point process defined by (1.2) is determinantal. The $(i, j)$-block of the correlation kernel is given by

$$
K_{i j}=W_{[i, k)} \Psi M^{-1} \Phi W_{[1, j)}-W_{[i, j)} .
$$

Proof. Take

$$
\mathfrak{X}=\{1, \ldots, n\} \cup \mathfrak{X}^{(1)} \cup \cdots \cup \mathfrak{X}^{(k)}
$$


and consider the conditional $L$-ensemble on $\mathfrak{X}$ with $\mathfrak{Y}=\mathfrak{X}^{(1)} \cup \cdots \cup \mathfrak{X}^{(k)}$ and the matrix $L$ given in the block form by

$$
L=\left[\begin{array}{cccccc}
0 & \Phi & 0 & 0 & \cdots & 0 \\
0 & 0 & -W_{1} & 0 & \cdots & 0 \\
0 & 0 & 0 & -W_{2} & \cdots & 0 \\
\cdots & \cdots & \cdots & \cdots & \cdots & \cdots \\
0 & 0 & 0 & 0 & \cdots & -W_{k-1} \\
\Psi & 0 & 0 & 0 & \cdots & 0
\end{array}\right]
$$

Then this conditional $L$-ensemble is exactly the point process defined by (1.2). Indeed, the determinant of a block matrix of type (1.5) is nonzero if and only if the sizes of all blocks are equal, and in that case the determinant is equal to the product of determinants of the nonzero blocks up to a sign which depends only on the size of the blocks. This observation immediately implies that (1.1) and (1.2) are equivalent.

According to Proposition 1.2, in order to compute the correlation kernel we need to invert $\mathbf{1}_{\mathfrak{Y}}+L$.

Lemma 1.5. The following inversion formula for a block matrix with square $(1,1)$ and (2,2) blocks holds:

$$
\left[\begin{array}{ll}
A & B \\
C & D
\end{array}\right]^{-1}=\left[\begin{array}{cc}
-\mathcal{M}^{-1} & \mathcal{M}^{-1} B D^{-1} \\
D^{-1} C \mathcal{M}^{-1} & D^{-1}-D^{-1} C \mathcal{M}^{-1} B D^{-1}
\end{array}\right], \quad \mathcal{M}=B D^{-1} C-A
$$

where we assume that all the needed inverses exist.

Proof. The matrix in the right-hand side equals

$$
\left[\begin{array}{cc}
1 & 0 \\
-D^{-1} C & 1
\end{array}\right]\left[\begin{array}{cc}
-\mathcal{M}^{-1} & \mathcal{M}^{-1} B D^{-1} \\
0 & D^{-1}
\end{array}\right]
$$

Inverting this product we obtain

$$
\left[\begin{array}{cc}
-\mathcal{M} & B \\
0 & D
\end{array}\right]\left[\begin{array}{cc}
1 & 0 \\
D^{-1} C & 1
\end{array}\right]=\left[\begin{array}{cc}
-\mathcal{M}+B D^{-1} C & B \\
C & D
\end{array}\right]=\left[\begin{array}{cc}
A & B \\
C & D
\end{array}\right]
$$

We now split $\mathbf{1}_{\mathfrak{Y}}+L$ into blocks according to the splitting $\mathfrak{X}=\{1, \ldots, n\} \cup \mathfrak{Y}$ and use the above lemma. First of all,

$$
D^{-1}=\left[\begin{array}{ccccc}
1 & -W_{1} & 0 & \cdots & 0 \\
0 & 1 & -W_{2} & \cdots & 0 \\
0 & 0 & 1 & \cdots & 0 \\
\cdots & \cdots & \cdots & \cdots & \cdots \\
0 & 0 & 0 & \cdots & 1
\end{array}\right]^{-1}=\left[\begin{array}{ccccc}
1 & W_{[1,2)} & W_{[1,3)} & \cdots & W_{[1, k)} \\
0 & 1 & W_{[2,3)} & \cdots & W_{[2, k)} \\
0 & 0 & 1 & \cdots & W_{[3, k)} \\
\cdots & \cdots & \cdots & \cdots & \cdots \\
0 & 0 & 0 & \cdots & 1
\end{array}\right]
$$

Next, $\mathcal{M}=B D^{-1} C-A=\Phi W_{[1, k)} \Psi$ is exactly the matrix $M$ given by (1.3). It readily follows that $\mathbf{1}_{\mathfrak{Y}}-\left(D^{-1}-D^{-1} C M^{-1} B D^{-1}\right)$ is exactly the right-hand side of (1.4).

We now aim at proving a Pfaffian analog of Theorem 1.4. In order to work with $2 \times 2$ matrix valued matrices, we introduce two copies of our (finite) phase space $\mathfrak{X}$ 
which we will denote by $\mathfrak{X}^{\prime}$ and $\mathfrak{X}^{\prime \prime}$. Each point $x \in \mathfrak{X}$ has a prototype $x^{\prime} \in \mathfrak{X}^{\prime}$ and another one $x^{\prime \prime} \in \mathfrak{X}^{\prime \prime}$.

A Pfaffian L-ensemble on $\mathfrak{X}$ is a random point process on $\mathfrak{X}$ with probabilities of the point configurations given by

$$
\operatorname{Prob}\{X\}=\frac{\operatorname{Pf} L_{X}}{\operatorname{Pf}(\mathbf{J}+L)}, \quad X \subset \mathfrak{X} .
$$

Here $L$ is a $|\mathfrak{X}| \times|\mathfrak{X}|$ skew-symmetric matrix made of $2 \times 2$ blocks with rows and columns parameterized by points of $\mathfrak{X}$. Alternatively, it is a $2|\mathfrak{X}| \times 2|\mathfrak{X}|$ matrix with rows and column parameterized by elements of $\mathfrak{X}^{\prime} \cup \mathfrak{X}^{\prime \prime}$. The $2 \times 2$ blocks have the form

$$
L(x, y)=\left[\begin{array}{ll}
L\left(x^{\prime}, y^{\prime}\right) & L\left(x^{\prime}, y^{\prime \prime}\right) \\
L\left(x^{\prime \prime}, y^{\prime}\right) & L\left(x^{\prime \prime}, y^{\prime \prime}\right)
\end{array}\right] .
$$

The matrix $\mathbf{J}$ is defined by

$$
\mathbf{J}(x, y)=\left\{\begin{array}{cc}
{\left[\begin{array}{cc}
0 & 1 \\
-1 & 0
\end{array}\right],} & x=y \\
0, & x \neq y .
\end{array}\right.
$$

A random point process is called Pfaffian if there exists a $2 \times 2$ matrix valued $|\mathfrak{X}| \times|\mathfrak{X}|$ skew-symmetric matrix $K$ with rows and column parameterized by points of $\mathfrak{X}$, such that the correlation functions of the process have the Pfaffian form: $\rho(Y)=\operatorname{Pf} K_{Y}$ for any $Y \subset \mathfrak{X}$. As in the determinantal case, the matrix $K$ is called the correlation kernel.

Similarly to Proposition 1.1, we have the following statement.

Proposition 1.6 $[\mathrm{R}]$. The Pfaffian L-ensemble as defined above is a Pfaffian point process with the correlation kernel $K=\mathbf{J}+(\mathbf{J}+L)^{-1}$.

Once again, let us take a subset $\mathfrak{Y}$ of $\mathfrak{X}$ and let us consider a new random point process on $\mathfrak{Y}$ by taking the intersections of the random point configuration of the Pfaffian $L$-ensemble with $\mathfrak{Y}$, provided that these configurations contain the complement $\overline{\mathfrak{Y}}=\mathfrak{X} \backslash \mathfrak{Y}$. Then the probabilities of the point configurations for such a process are given by

$$
\operatorname{Prob}\{Y\}=\frac{\operatorname{Pf} L_{Y \cup \bar{Y}}}{\operatorname{Pf}\left(\mathbf{J}_{\mathfrak{Y}}+L\right)}, \quad Y \subset \mathfrak{Y} .
$$

We call this process the conditional Pfaffian L-ensemble. Proposition 1.6 above is a corollary of the following more general claim, cf. Proposition 1.2.

Proposition 1.7. The conditional Pfaffian L-ensemble is a Pfaffian point process. Its correlation kernel is given by

$$
K=\mathbf{J}_{\mathfrak{Y}}+\left.\left(\mathbf{J}_{\mathfrak{Y}}+L\right)^{-1}\right|_{\mathfrak{Y} \times \mathfrak{Y}} .
$$

Proof. We have

$$
\operatorname{Pf} K_{Y}=\sum_{X \subset Y} \operatorname{Pf}\left(\left(\mathbf{J}_{\mathfrak{Y}}+L\right)^{-1}\right)_{X}=\sum_{Z=\bar{X} \supset \bar{Y}}(-1)^{|X|} \frac{\operatorname{Pf}\left(\mathbf{J}_{\mathfrak{Y}}+L\right)_{Z}}{\operatorname{Pf}\left(\mathbf{J}_{\mathfrak{Y}}+L\right)},
$$


and the rest is as in the proof of Proposition 1.2. Here we used the following fact: if $A$ and $B$ are $2 l \times 2 l$ skew-symmetric matrices and $B=A^{-1}$ then

$$
\operatorname{Pf} A_{\alpha_{1}, \ldots, \alpha_{2 m}}=(-1)^{\alpha_{1}+\cdots+\alpha_{2 m}} \cdot \frac{\operatorname{Pf} B_{\{1, \ldots, 2 l\} \backslash\left\{\alpha_{1}, \ldots, \alpha_{2 m}\right\}}}{\operatorname{Pf} B} .
$$

We proceed to stating the Pfaffian analog of the Eynard-Mehta theorem. Let us assume that our state space is a union of $k$ subsets $\mathfrak{X}^{(1)} \cup \cdots \cup \mathfrak{X}^{(k)}$, and consider a random point process that lives on $2 n k$ point configurations with exactly $2 n$ points in each $\mathfrak{X}^{(i)}, i=1, \ldots, k$. The probability of any such point configuration is given by

$$
\begin{aligned}
& \operatorname{Prob}\left\{\left\{x_{i}^{(1)}\right\}_{i=1}^{2 n} \cup \cdots \cup\left\{x_{i}^{(k)}\right\}_{i=1}^{2 n}\right\} \\
&=\text { const } \cdot \operatorname{Pf}_{1 \leq i, j \leq 2 n}\left[\epsilon\left(x_{i}^{(1)}, x_{j}^{(1)}\right)\right] \cdot \operatorname{det}_{1 \leq i, j \leq 2 n}\left[V_{1}\left(x_{i}^{(1)}, x_{j}^{(2)}\right)\right] \cdots \\
& \quad \cdots \operatorname{det}_{1 \leq i, j \leq 2 n}\left[V_{k-1}\left(x_{i}^{(k-1)}, x_{j}^{(k)}\right)\right]_{1 \leq i, j \leq 2 n} \operatorname{det}_{1}\left[\xi_{i}\left(x_{j}^{(k)}\right)\right] .
\end{aligned}
$$

Here $\left\{\xi_{i}\right\}_{i=1, \ldots, 2 n}$, are some functions on $\mathfrak{X}^{(k)},\left\{V_{m}\right\}_{m=1, \ldots, k-1}$, are matrices with rows parameterized by points of $\mathfrak{X}^{(m)}$ and columns parameterized by points of $\mathfrak{X}^{(m+1)}$, and $\epsilon$ is a skew-symmetric matrix with rows and columns parameterized by the points of $\mathfrak{X}^{(1)}$.

As before, it is convenient to organize $\xi_{i}$ 's into one $\left|\mathfrak{X}^{(k)}\right| \times 2 n$ matrix $\Xi$ with columns parameterized by $1, \ldots, 2 n$, and rows parameterized by $\mathfrak{X}^{(k)}$; the matrix elements are the values $\xi_{i}\left(x^{(k)}\right), x^{(k)} \in \mathfrak{X}^{(k)}$.

The next statement is an analog of Lemma 1.3.

Lemma 1.8. The sum of the right-hand sides of (1.2) with "const" removed, taken over all possible point configurations is equal to $\operatorname{Pf} N$, where

$$
N=\Xi^{t} V_{k-1}^{t} \cdots V_{1}^{t} \epsilon V_{1} \cdots V_{k-1} \Xi .
$$

Thus, const in (1.6) is equal to $\operatorname{Pf} N^{-1}$, provided that $\operatorname{Pf} N \neq 0$.

Using the familiar notation

$$
V_{[i, j)}= \begin{cases}V_{i} \cdots V_{j-1}, & i<j \\ 0, & i \geq j\end{cases}
$$

we have $N=\Xi^{t} V_{[1, k)}^{t} \epsilon V_{[1, k)} \Xi$. In what follows, we will always assume that this matrix is nondegenerate.

Theorem 1.9. The random point process defined by (1.6) is Pfaffian. The $2 \times 2$ entries of the correlation kernel in its $(i, j)$-block are given by

$$
\left[\begin{array}{cc}
V_{[i, k)} \Xi N^{-1} \Xi^{t} V_{[j, k)}^{t} & V_{[i, k)} \Xi N^{-1} \Xi^{t} V_{[1, k)}^{t} \epsilon V_{[1, j)}-V_{[i, j)} \\
-V_{[1, i)}^{t} \epsilon V_{[1, k)} \Xi N^{-1} \Xi^{t} V_{[j, k)}^{t}+V_{[j, i)}^{t} & -V_{[1, i)}^{t} \epsilon V_{[1, k)} \Xi N^{-1} \Xi^{t} V_{[1, k)}^{t} \epsilon V_{[1, j)}+V_{[1, i)}^{t} \epsilon V_{[1, j)}
\end{array}\right]
$$

Proof. Take

$$
\mathfrak{X}=\{1, \ldots, 2 n\} \cup \mathfrak{X}^{(1)} \cup \cdots \cup \mathfrak{X}^{(k)}
$$


and consider the conditional Pfaffian $L$-ensemble on $\mathfrak{X}$ with $\mathfrak{Y}=\mathfrak{X}^{(1)} \cup \cdots \cup \mathfrak{X}^{(k)}$ and the matrix $L$ which in the block form corresponding to the splitting

$$
\{1, \ldots, 2 n\} \cup\left(\mathfrak{X}^{(1)}\right)^{\prime} \cup\left(\mathfrak{X}^{(1)}\right)^{\prime \prime} \cup \cdots\left(\mathfrak{X}^{(k)}\right)^{\prime} \cup\left(\mathfrak{X}^{(k)}\right)^{\prime \prime}
$$

has the form

$$
L=\left[\begin{array}{cccccccccc}
0 & 0 & 0 & 0 & 0 & 0 & \cdots & 0 & 0 & \Xi^{t} \\
0 & \epsilon & 0 & 0 & 0 & 0 & \cdots & 0 & 0 & 0 \\
0 & 0 & 0 & V_{1} & 0 & 0 & \cdots & 0 & 0 & 0 \\
0 & 0 & -V_{1}^{t} & 0 & 0 & 0 & \cdots & 0 & 0 & 0 \\
0 & 0 & 0 & 0 & 0 & V_{2} & \cdots & 0 & 0 & 0 \\
0 & 0 & 0 & 0 & -V_{2}^{t} & 0 & \cdots & 0 & 0 & 0 \\
\cdots & \cdots & \cdots & \cdots & \cdots & \cdots & \cdots & \cdots & \cdots & \cdots \\
0 & 0 & 0 & 0 & 0 & 0 & \cdots & 0 & V_{k-1} & 0 \\
0 & 0 & 0 & 0 & 0 & 0 & \cdots & -V_{k-1}^{t} & 0 & 0 \\
-\Xi & 0 & 0 & 0 & 0 & 0 & \cdots & 0 & 0 & 0
\end{array}\right]
$$

Then this conditional Pfaffian $L$-ensemble is exactly the process defined by (1.6). We want to use Proposition 1.7 and Lemma 1.5. Writing $\left(\mathbf{J}_{\mathfrak{Y}}+L\right)$ in $2 \times 2$ block form corresponding to the splitting

$$
\{1, \ldots, 2 n\} \cup\left(\left(\mathfrak{X}^{(1)}\right)^{\prime} \cup\left(\mathfrak{X}^{(1)}\right)^{\prime \prime} \cup \cdots\left(\mathfrak{X}^{(k)}\right)^{\prime} \cup\left(\mathfrak{X}^{(k)}\right)^{\prime \prime}\right)
$$

and using the notation of Lemma 1.5, we obtain that the $(i, j)$-block of $\left(\mathbf{J}+D^{-1}\right)$ has the form

$$
\left[\begin{array}{cc}
0 & -V_{[i, j)} \\
V_{[j, i)}^{t} & V_{[1, i)}^{t} \epsilon V_{[1, j)}
\end{array}\right]
$$

This follows, for example, from the explicit computation of the terminating series

$$
D^{-1}=\left(\mathbf{J}_{\mathfrak{Y}}+L_{\mathfrak{Y}}\right)^{-1}=-\mathbf{J}_{\mathfrak{Y}}\left(\mathbf{1}+L_{\mathfrak{Y}} \mathbf{J}_{\mathfrak{Y}}+\left(L_{\mathfrak{Y}} \mathbf{J}_{\mathfrak{Y}}\right)^{2}+\cdots+\left(L_{\mathfrak{Y}} \mathbf{J}_{\mathfrak{Y}}\right)^{2 k-1}\right)
$$

Further,

$$
\begin{gathered}
\mathcal{M}=-\Xi^{t} V_{[1, k)} \epsilon V_{[1, k)} \Xi=-N, \\
D^{-1} C=\left[V_{[1, k)} \Xi,-\epsilon V_{[1, k)} \Xi, V_{[2, k)} \Xi,-V_{[1,2)}^{t} \epsilon V_{[1, k)} \Xi, \ldots, \Xi,-V_{[1, k)}^{t} \epsilon V_{[1, k)} \Xi\right]^{t}, \\
B D^{-1}=\left[\Xi^{t} V_{[1, k)}^{t}, \Xi^{t} V_{[1, k)}^{t} \epsilon, \Xi^{t} V_{[2, k)}^{t}, \Xi^{t} V_{[1, k)}^{t} \epsilon V_{[1,2)}, \ldots, \Xi^{t}, \Xi^{t} V_{[1, k)}^{t} \epsilon V_{[1, k)},\right]
\end{gathered}
$$

and the $(i, j)$-block of

$$
\mathbf{J}_{Y}+\left.\left(\mathbf{J}_{\mathfrak{Y}}+L\right)^{-1}\right|_{\mathfrak{Y} \times \mathfrak{Y}}=\mathbf{J}_{\mathfrak{Y}}+\left(D^{-1}-D^{-1} C \mathcal{M}^{-1} B D^{-1}\right)
$$

is readily seen to be equal to (1.7). 


\section{SCHUR PROCESS}

In the next two sections we will be extensively using the theory of symmetric functions; we refer the reader to the book $[\mathrm{M}]$ which contains all needed notations and definitions.

Pick a natural number $T$ and consider all sequences of partitions (equivalently, Young diagrams) of the form

$$
\varnothing \subset \lambda^{(1)} \supset \mu^{(1)} \subset \lambda^{(2)} \supset \mu^{(2)} \subset \cdots \supset \mu^{(T-1)} \subset \lambda^{(T)} \supset \varnothing .
$$

To any such sequence we assign the weight

$$
\mathcal{W}(\lambda, \mu)=s_{\lambda^{(1)}}\left(\rho_{0}^{+}\right) s_{\lambda^{(1)} / \mu^{(1)}}\left(\rho_{1}^{-}\right) s_{\lambda^{(2)} / \mu^{(1)}}\left(\rho_{1}^{+}\right) \cdots s_{\lambda^{(T)} / \mu^{(T-1)}}\left(\rho_{T-1}^{+}\right) s_{\lambda^{(T)}}\left(\rho_{T}^{-}\right) .
$$

In this formula, there is one factor for any two neighboring partitions in the sequence. All of the factors, except for the first and the last ones, are of the form $s_{\lambda / \mu}(\rho)$. The $\rho$ 's here are specializations of the algebra $\Lambda$ of symmetric functions, $s_{\lambda}$ 's are the Schur functions, and $s_{\lambda / \mu}$ 's are the skew Schur functions.

We will use the notation $l_{i}=\lambda_{i}-i, m_{i}=\mu_{i}-i$. Note that $s_{\lambda / \mu}$ can be written as a determinant of a submatrix of the Toeplitz matrix $\left[h_{i-j}\right]$ :

$$
s_{\lambda / \mu}=\operatorname{det}\left[h_{l_{i}-m_{j}}\right]_{i, j=1}^{N}, \quad N \geq \max \{l(\lambda), l(\mu)\} .
$$

Here $h_{i}$ 's are the complete homogeneous symmetric functions, and $h_{i}=0$ if $i<0$. Their generating function will be denoted by

$$
\sum_{k \geq 0} h_{k}(\rho) z^{k}=H(\rho ; z)
$$

We will use the notation

$$
H\left(\rho^{\prime} ; \rho^{\prime \prime}\right)=\sum_{\lambda} s_{\lambda}\left(\rho^{\prime}\right) s_{\lambda}\left(\rho^{\prime \prime}\right)
$$

If $\rho^{\prime}$ and $\rho^{\prime \prime}$ are specializations into sets of variables $x, y$ then one has the Cauchy identity

$$
H(x ; y)=\prod_{i, j}\left(1-x_{i} y_{j}\right)^{-1} .
$$

Both sides of this identity should be viewed as formal series with elements from $\Lambda \otimes \Lambda$; these series "converge" in the sense that there are only finitely many terms of any fixed degree. In what follows we will usually omit comments of the same kind.

For two specializations $\rho^{\prime}$ and $\rho^{\prime \prime}$ we denote by $\rho^{\prime} \cup \rho^{\prime \prime}$ the specialization which adds the power sums:

$$
p_{k}\left(\rho^{\prime} \cup \rho^{\prime \prime}\right)=p_{k}\left(\rho^{\prime}\right)+p_{k}\left(\rho^{\prime \prime}\right), \quad k \geq 1
$$


Proposition 2.1. The sum of the weights (2.2) over all sequences (2.1) is equal to

$$
Z(\rho)=\prod_{0 \leq i<j \leq T} H\left(\rho_{i}^{+} ; \rho_{j}^{-}\right)
$$

Proof. Follows from the well known identity, see [M, I.5.26],

$$
\sum_{\nu} s_{\nu / \lambda}(x) s_{\nu / \mu}(y)=H(x ; y) \sum_{\tau} s_{\mu / \tau}(x) s_{\lambda / \tau}(y) .
$$

Using this formula to sum (2.2) over all $\lambda^{(i)}$ reduces the statement to a similar one with smaller length $T$ of the sequence (2.1). Induction on $T$ completes the proof.

We now consider a (formal) random point process on $\{1, \ldots, T\} \times \mathbb{Z}$ by assigning to a sequence $(2.1)$ the point configuration

$$
\mathcal{L}(\lambda)=\left\{\left(1, \lambda_{i}^{(1)}-i\right)\right\}_{i \geq 1} \cup \cdots \cup\left\{\left(T, \lambda_{i}^{(T)}-i\right)\right\}_{i \geq 1} .
$$

The "probability" of this point configuration is given by the weight (2.2) divided by $Z(\rho)$. The correlation functions of this point process are given by the following statement.

Theorem 2.2 (Okounkov-Reshetikhin $[\mathrm{OR}]$ ). The random point process defined above is determinantal. In other words, for any pairwise distinct points $\left(i_{s}, u_{s}\right), 1 \leq s \leq S$, of $\{1, \ldots, T\} \times \mathbb{Z}$ we have the following formal series identity

$$
\sum_{\left\{\left(i_{1}, u_{1}\right), \ldots,\left(i_{S}, u_{S}\right)\right\} \subset \mathcal{L}(\lambda)} \mathcal{W}(\lambda, \mu)=Z(\rho) \cdot \operatorname{det}_{1 \leq s, t \leq S}\left[K\left(i_{s}, u_{s} ; i_{t}, u_{t}\right)\right]
$$

where

$$
K(i, u ; j, v)=\frac{1}{(2 \pi i)^{2}} \oint \oint \frac{H\left(\rho_{[i, T]}^{-} ; z\right) H\left(\rho_{[0, j)}^{+} ; w\right)}{(z w-1) H\left(\rho_{[0, i)}^{+} ; z^{-1}\right) H\left(\rho_{[j, T]}^{-} ; w^{-1}\right)} \frac{d z d w}{z^{u+1} w^{v+1}}
$$

The contours for $z$ and $w$ go around 0 in the positive direction so that for $i \leq j$ we take $|z|>1,|w|>1$ meaning that we may expand

$$
(z w-1)^{-1}=(z w)^{-1}+(z w)^{-2}+\ldots
$$

to evaluate the kernel, while for $i \geq j$ we take $|z|<1,|w|<1$ thus allowing the expansion

$$
(z w-1)^{-1}=-\left(1+z w+(z w)^{2}+\ldots\right) .
$$

Remark 2.3. As will be shown in the proof, (2.6)-(2.7) becomes a numeric equality for arbitrary finite dimensional specializations $\rho^{ \pm}$with values of the variables taken from the open unit disc, and contours in (2.7) taken close enough to the unit circle. By a simple approximation argument it follows that (2.6)-(2.7) holds for arbitrary specializations $\rho^{ \pm}$such that the radii of convergence of $H\left(\rho_{i}^{ \pm} ; z\right)$ are strictly greater 
than 1, and the contours are chosen close enough to the unit circle. As was shown by Johansson [J2], these analytic restrictions can be further relaxed.

Proof. It suffices to prove (2.6) when $\rho_{0}^{+}$and $\rho_{T}^{-}$are specializations into finitely many variables:

$$
\rho_{0}^{+}=\left(x_{1}, \ldots, x_{p}\right), \quad \rho_{T}^{-}=\left(y_{1}, \ldots, y_{p}\right) .
$$

If we sum (2.2) over all $\mu^{(i)}$ 's with $\lambda^{(j)}$ 's fixed, use (2.3) and the definition of the Schur polynomial as a ratio of two determinants, see [M,I.3(3.1)], we obtain

$$
\begin{array}{r}
\frac{\prod_{i=1}^{p}\left(x_{i} y_{i}\right)^{p}}{\prod_{1 \leq i<j \leq p}\left(x_{i}-x_{j}\right)\left(y_{i}-y_{j}\right)} \cdot \operatorname{det}_{1 \leq i, j \leq p}\left[x_{i}^{l_{j}^{(1)}}\right] \operatorname{det}_{1 \leq i, j \leq N} W_{1}\left(l_{i}^{(1)}, l_{j}^{(2)}\right) \cdots \\
\cdots \operatorname{det}_{1 \leq i, j \leq N} W_{T-1}\left(l_{i}^{(T-1)}, l_{j}^{(T)}\right) \operatorname{det}_{1 \leq i, j \leq p}\left[y_{i}^{l_{j}^{(T)}}\right]
\end{array}
$$

where $N$ is large enough, $N \geq \max \left\{l\left(\lambda^{(i)}\right)\right\}$, and $\left\|W_{i}(x, y)\right\|_{x, y \in \mathbb{Z}}$ are Toeplitz matrices with symbols

$$
\sum_{m \in \mathbb{Z}} W_{i}(x+m, x) z^{m}=H\left(\rho_{i}^{-} ; z\right) H\left(\rho_{i}^{+} ; z^{-1}\right) .
$$

The formula (2.8) is very similar to (1.2). There are two important differences though: the intermediate determinants in (2.8) may be of any finite size $N$, and the variables $l_{j}^{(i)}$ may vary over the infinite set of all integers, not over some finite set $\mathfrak{X}$.

However, if we are interested only in the terms of (2.2) of a small enough degree, we may restrict our attention to Young diagrams $\lambda^{(i)}$ with bounded lengths of the first row and column, which translates into boundedness of $l\left(\lambda^{(i)}\right)$ and $l_{j}^{(i)}$. Thus, in order to correctly evaluate the terms of (2.2) of a fixed degree we may choose $p$ large enough and assume that in (2.8), $N=p$ and $l_{j}^{(i)}$ 's vary in a finite set. Therefore, we are in a position to apply Theorem 1.4.

The hard part in the application of Theorem 1.4 is the computation of $M^{-1}$. Thanks to (1.3) and (2.4), we know that up to terms of high degree

$$
\begin{aligned}
& \frac{\prod_{i=1}^{p}\left(x_{i} y_{i}\right)^{p}}{\prod_{1 \leq i<j \leq p}\left(x_{i}-x_{j}\right)\left(y_{i}-y_{j}\right)} \operatorname{det} M=\prod_{0 \leq i<j \leq T} H\left(\rho_{i}^{+} ; \rho_{j}^{-}\right) \\
& \quad=\prod_{i=1}^{p} H\left(\rho_{[1, T-1]}^{-} ; x_{i}\right) H\left(\rho_{[1, T-1]}^{+} ; y_{i}\right) \cdot \prod_{i, j=1}^{p} \frac{1}{1-x_{i} y_{j}} \cdot \prod_{1 \leq i<j \leq T-1} H\left(\rho_{i}^{+} ; \rho_{j}^{-}\right),
\end{aligned}
$$

where we use the notation $\rho_{[i, j]}^{ \pm}=\rho_{i}^{ \pm} \cup \rho_{i+1}^{ \pm} \cup \cdots \cup \rho_{j}^{ \pm}$.

On the other hand, it is not hard to see that computing the determinant of $M$ with $k$ th row and $l$ th column removed is, up to terms of high degree, equivalent to repeating the above computation with variables $x_{k}$ and $y_{l}$ removed from the 
specializations $\rho_{0}^{+}$and $\rho_{T}^{-}$:

$$
\begin{aligned}
& \frac{\left(x_{1} \cdots \hat{x}_{k} \cdots x_{p} y_{1} \cdots \hat{y}_{l} \cdots y_{p}\right)^{p}}{\prod_{1 \leq i<j \leq p, i \neq k, j \neq l}\left(x_{i}-x_{j}\right)\left(y_{i}-y_{j}\right)} \operatorname{det} M\left(\begin{array}{c}
1 \cdots \hat{k} \cdots p \\
1 \cdots \hat{l} \cdots p
\end{array}\right) \\
& =\prod_{i=1}^{p} H\left(\rho_{[1, T-1]}^{-} ; x_{i}\right) H\left(\rho_{[1, T-1]}^{+} ; y_{i}\right) \cdot \prod_{i, j=1}^{p} \frac{1}{1-x_{i} y_{j}} \cdot \prod_{1 \leq i<j \leq T-1} H\left(\rho_{i}^{+} ; \rho_{j}^{-}\right) \\
& \times \frac{\prod_{i=1}^{p}\left(1-x_{k} y_{i}\right)\left(1-x_{i} y_{l}\right)}{H\left(\rho_{[1, T-1]}^{-} ; x_{k}\right) H\left(\rho_{[1, T-1]}^{+} ; y_{l}\right)\left(1-x_{k} y_{l}\right)} .
\end{aligned}
$$

The conclusion is that up to terms of high degree,

$$
\begin{aligned}
& \left(M^{-1}\right)_{l k}=\frac{(-1)^{k+l} \operatorname{det} M\left(\begin{array}{c}
1 \cdots \hat{k} \cdots p \\
1 \ldots \hat{l} \cdots p
\end{array}\right)}{\operatorname{det} M} \\
& =\frac{x_{k} y_{l}}{\prod_{i \neq k}\left(1-x_{i} / x_{k}\right) \prod_{j \neq l}\left(1-y_{j} / y_{l}\right)} \frac{\prod_{i=1}^{p}\left(1-x_{k} y_{i}\right)\left(1-x_{i} y_{l}\right)}{H\left(\rho_{[1, T-1]}^{-} ; x_{k}\right) H\left(\rho_{[1, T-1]}^{+} ; y_{l}\right)\left(1-x_{k} y_{l}\right)} .
\end{aligned}
$$

Hence, in the notation of (1.4) we have

$$
\begin{aligned}
& \left(\Psi M^{-1} \Phi\right)_{u v} \\
= & \sum_{k, l=1}^{p} \frac{x_{k}^{v+1} y_{l}^{u+1}}{\prod_{i \neq k}\left(1-x_{i} / x_{k}\right) \prod_{j \neq l}\left(1-y_{j} / y_{l}\right)} \frac{\prod_{i=1}^{p}\left(1-x_{k} y_{i}\right)\left(1-x_{i} y_{l}\right)}{H\left(\rho_{[1, T-1]}^{-} ; x_{k}\right) H\left(\rho_{[1, T-1]}^{+} ; y_{l}\right)\left(1-x_{k} y_{l}\right)} \\
& =\frac{1}{(2 \pi i)^{2}} \oint \oint \frac{H\left(\rho_{0}^{+} ; z^{-1}\right) H\left(\rho_{T}^{-} ; w^{-1}\right) z^{v} w^{u}}{(1-z w) H\left(\rho_{[1, T]}^{-} ; z\right) H\left(\rho_{[0, T)}^{+} ; w\right)} d z d w .
\end{aligned}
$$

The last equality is just a formal evaluation of residues of the integrand at the points $z=x_{k}, w=y_{l} ; k, l=1, \ldots, p$. Then, using the same rule of evaluating the integrals, up to terms of high degree, we obtain

$$
\left(W_{[i, k)} \Psi M^{-1} \Phi W_{[i, j)}\right)_{u v}=\frac{1}{(2 \pi i)^{2}} \oint \oint \frac{H\left(\rho_{[0, j)}^{+} ; z^{-1}\right) H\left(\rho_{[i, T]}^{-} ; w^{-1}\right) z^{v} w^{u}}{(1-z w) H\left(\rho_{[j, T]}^{-} ; z\right) H\left(\rho_{[0, i)}^{+} ; w\right)} d z d w .
$$

Finally, if for $i<j$ we evaluate the residue of the right-hand side at $w=z^{-1}$, we get

$$
\left.-\frac{1}{2 \pi i} \oint H\left(\rho_{[i, j)}^{-} ; z\right) H\left(\rho_{[i, j)}^{+} ; z^{-1}\right) z^{v-u-1} d z=\left(-W_{[i, j}\right)\right)_{u v} .
$$

Thus, (1.4) implies the statement of the theorem ${ }^{2}$ for finite-dimensional specializations $\rho_{0}^{+}=\left(x_{1}, \ldots, x_{p}\right), \rho_{T}^{-}=\left(y_{1}, \ldots, y_{p}\right)$, with the following (formal) rule of evaluating the double contour integral: for $i \leq j$ we sum up all the residues at $z=x_{k}, w=y_{l}$, and for $i>j$ we also add the residue at $w=z^{-1}$.

If we now assume that all our specializations $\rho_{i}^{ \pm}$are finite-dimensional with numeric values of the variables taken from the open unit disc, then this evaluation rule will give the actual value of the integral if for $i \leq j$ we take the contours to be circles $|z|=|w|=1-\varepsilon$ with small enough $\varepsilon>0$, and for $i>j$ we take the circles $|z|=|w|=1+\varepsilon$ with small enough $\varepsilon>0$. Thus, in this case we can evaluate the integral in a different way, by expanding $(1-z w)^{-1}$ and all the $H$ 's into Taylor series and computing the residue at $z=0, w=0$. This proves our theorem for any finite dimensional specializations, and hence for any specializations.

\footnotetext{
${ }^{2}$ with the change $(z, w) \rightarrow\left(w^{-1}, z^{-1}\right)$ of the integration variables
} 


\section{Pfaffian Schur Process}

Once again, we consider sequences of Young diagrams of the form (2.1), but the weight $(2.2)$ is replaced by

$$
\mathcal{V}(\lambda, \mu)=\tau_{\lambda^{(1)}}\left(\rho_{0}^{+}\right) s_{\lambda^{(1)} / \mu^{(1)}}\left(\rho_{1}^{-}\right) s_{\lambda^{(2)} / \mu^{(1)}}\left(\rho_{1}^{+}\right) \cdots s_{\lambda^{(n)} / \mu^{(n-1)}}\left(\rho_{n-1}^{+}\right) s_{\lambda^{(n)}}\left(\rho_{n}^{-}\right)
$$

where the symmetric functions $\tau_{\lambda}$ are defined by

$$
\tau_{\lambda}=\sum_{\kappa^{\prime} \text { is even }} s_{\lambda / \kappa}
$$

Lemma 3.1. The symmetric function $\tau_{\lambda}$ can be written as a Pfaffian of a Toeplitz matrix made of complete homogeneous symmetric functions as follows:

$$
\tau_{\lambda}=\operatorname{Pf}\left[\sum_{a \in \mathbb{Z}}\left(h_{l_{i}-a-1} h_{l_{j}-a}-h_{l_{i}-a} h_{l_{j}-a-1}\right)\right]_{1 \leq i, j \leq 2 N}, \quad l(\lambda) \leq 2 N .
$$

Proof. It is not hard to see that the indicator function for partitions $\kappa$ with even conjugate and $l(\mu) \leq 2 N$ can be expressed as a Pfaffian:

$$
\chi(\kappa)=\operatorname{Pf}_{1 \leq i, j \leq 2 N}\left[\delta_{\kappa_{i}-i-1, \kappa_{j}-j}-\delta_{\kappa_{i}-i, \kappa_{j}-j-1}\right] .
$$

Using the Pfaffian variant of the Cauchy-Binet formula and the notation $k_{i}=\kappa_{i}-i$, we obtain (all determinants/Pfaffians are of size $2 N \geq l(\lambda)$ )

$$
\begin{aligned}
\tau_{\lambda}=\sum_{\kappa} \operatorname{det}\left[h_{l_{i}-k_{j}}\right] \operatorname{Pf}\left[\delta_{\kappa_{i}-i-1, \kappa_{j}-j}-\delta_{\kappa_{i}-i, \kappa_{j}-j-1}\right] \\
=\sum_{\kappa} \operatorname{Pf}\left[\left\|h_{l_{i}-k_{j}}\right\| \cdot\left\|\delta_{\kappa_{i}-i-1, \kappa_{j}-j}-\delta_{\kappa_{i}-i, \kappa_{j}-j-1}\right\| \cdot\left\|h_{l_{i}-k_{j}}\right\|^{t}\right] \\
=\operatorname{Pf}\left[\sum_{a \in \mathbb{Z}}\left(h_{l_{i}-a-1} h_{l_{j}-a}-h_{l_{i}-a} h_{l_{j}-a-1}\right)\right] .
\end{aligned}
$$

The definition of $\tau_{\lambda}$ implies that if we specialize $\tau_{\lambda}$ into one nonzero variable $\alpha$ then $\tau_{\lambda}(\alpha)=\alpha^{\sum_{i \geq 1} \lambda_{2 i-1}-\lambda_{2 i}}$ (there is a unique choice of $\kappa$ that gives a nonzero contribution). In particular, $\tau_{\lambda}(1)=1$.

Note also that the symbol of the Toeplitz matrix in (3.2) is equal to

$$
\left(z^{-1}-z\right) H(\rho ; z) H\left(\rho ; z^{-1}\right) .
$$

In addition to the notation $H\left(\rho^{\prime} ; \rho^{\prime \prime}\right)$ introduced in the previous section, we define

$$
H^{o}(\rho)=\sum_{\lambda^{\prime} \text { is even }} s_{\lambda}(\rho) .
$$

If $\rho$ is the specialization into a set of variables $x$ then

$$
H^{o}(x)=\prod_{i<j}\left(1-x_{i} x_{j}\right)^{-1} .
$$

We have the following analog of Proposition 2.1. 
Proposition 3.2. The sum of weights (3.1) over all sequences (2.1) is equal to

$$
Z^{o}(\rho)=H^{o}\left(\rho_{[1, T]}^{-}\right) \prod_{0 \leq i<j \leq T} H\left(\rho_{i}^{+} ; \rho_{j}^{-}\right)
$$

Proof. As in the proof of Proposition 2.1, we sum over all $\lambda^{(i)}$ using the identity used there together with, see [M, I.5.27],

$$
\sum_{\nu^{\prime} \text { even }} s_{\nu / \lambda}(x)=H^{o}(x) \sum_{\kappa^{\prime} \text { even }} s_{\lambda / \kappa}
$$

thus reducing the statement to the case of smaller $T$.

Similarly to $\S 2$, we consider the random point process on $\{1, \ldots, T\} \times \mathbb{Z}$ generated by the point configurations $\mathcal{L}(\lambda)$, see (2.5), and weights (3.1).

Theorem 3.3. The point process introduced above is Pfaffian. In other words, for any pairwise distinct points $\left(i_{s}, u_{s}\right), 1 \leq s \leq S$, of $\{1, \ldots, T\} \times \mathbb{Z}$ we have the following formal series identity

$$
\sum_{\left\{\left(i_{1}, u_{1}\right), \ldots,\left(i_{S}, u_{S}\right)\right\} \subset \mathcal{L}(\lambda)} \mathcal{V}(\lambda, \mu)=Z^{o}(\rho) \cdot \operatorname{Pf}\left[K\left(i_{s}, u_{s} ; i_{t}, u_{t}\right)\right]_{1 \leq s, t \leq S}
$$

where $K(i, u ; j, v)$ is a $2 \times 2$ matrix kernel

$$
K(i, u ; j, v)=\left[\begin{array}{ll}
K_{11}(i, u ; j, v) & K_{12}(i, u ; j, v) \\
K_{21}(i, u ; j, v) & K_{22}(i, u ; j, v)
\end{array}\right]
$$

whose blocks are given by:

$$
\begin{aligned}
& K_{11}(i, u ; j, v)=\frac{1}{(2 \pi i)^{2}} \\
\times & \iint \frac{(z-w)}{\left(z^{2}-1\right)\left(w^{2}-1\right)(z w-1)} \frac{H\left(\rho_{[i, T]}^{-} ; z\right) H\left(\rho_{[j, T]}^{-} ; w\right)}{H\left(\rho_{[1, T]}^{-} \cup \rho_{[0, i)}^{+} ; z^{-1}\right) H\left(\rho_{[1, T]}^{-} \cup \rho_{[0, j)}^{+} ; w^{-1}\right)} \frac{d z d w}{z^{u} w^{v}}
\end{aligned}
$$

The integrals are taken along closed contours which go around zero in the positive direction, and such that $|z|>1,|w|>1,{ }^{3}$

$$
\begin{aligned}
& K_{12}(i, u ; j, v)=-K_{21}(j, v ; i, u) \\
& \quad=\frac{1}{(2 \pi i)^{2}} \iint \frac{(z-w)}{\left(z^{2}-1\right)(z w-1) w} \frac{H\left(\rho_{[i, T]}^{-} ; z\right) H\left(\rho_{[1, T]}^{-} \cup \rho_{[0, j)}^{+} ; w\right)}{H\left(\rho_{[1, T]}^{-} \cup \rho_{[0, i)}^{+} ; z^{-1}\right) H\left(\rho_{[j, T]}^{-} ; w^{-1}\right)} \frac{d z d w}{z^{u} w^{v}}
\end{aligned}
$$

\footnotetext{
${ }^{3}$ This condition means that we may use the expansions

$$
\left(z^{2}-1\right)^{-1}=\sum_{k \geq 0} z^{-2 k-2}, \quad\left(w^{2}-1\right)^{-1}=\sum_{k \geq 0} w^{-2 k-2}, \quad(z w-1)^{-1}=\sum_{k \geq 0}(z w)^{-k-1}
$$
}

to see that this integral is a formal series of symmetric functions. Similar comments apply to other integral below. 
The integrals are taken along closed contours which go around zero in the positive direction, and such that $|z|>1$ and

- if $i \geq j$ then $|z w|>1$;

- if $i<j$ then $|z w|<1$.

Finally,

$$
\begin{aligned}
& K_{22}(i, u ; j, v)= \\
& \quad=\frac{1}{(2 \pi i)^{2}} \iint \frac{z-w}{z w(1-z w)} \frac{H\left(\rho_{[1, T]}^{-} \cup \rho_{[0, i)}^{+} ; z\right) H\left(\rho_{[1, T]}^{-} \cup \rho_{[0, j)}^{+} ; w\right)}{H\left(\rho_{[i, T]}^{-} ; z^{-1}\right) H\left(\rho_{[j, T]}^{-} ; w^{-1}\right)} \frac{d z d w}{z^{u} w^{v}}
\end{aligned}
$$

The integrals are taken along closed contours which go around zero in the positive direction, and such that $|z w|<1$.

Remark 3.4. Similarly to the determinantal case of $\S 2$, the statement of Theorem 3.3 becomes a numeric equality if all the specializations are such that the radii of convergence of $H\left(\rho_{i}^{ \pm} ; z\right)$ are strictly greater than 1 and the contours are chosen close enough to the unit circle.

Proof. Since the computations are very similar to those in the proof of Theorem 2.2 , we will omit the necessary justifications and just produce the formulas.

Using the similarity of (3.1) and (1.6), we will compute the correlation kernel via Theorem 1.9. Let us take $\rho_{T}^{-}$to be the finite dimensional specialization into variables $x_{1}, \ldots, x_{2 p}$. The the matrix $N^{-1}$ is computed using (3.3) in the same way as $M^{-1}$ in the proof of Theorem 2.2 was computed using (2.4). Namely, up to terms of high degree,

$$
\begin{aligned}
& \frac{\left(x_{1} \cdots x_{2 p}\right)^{2 p}}{\prod_{1 \leq i<j \leq 2 p}\left(x_{i}-x_{j}\right)} \operatorname{Pf} N=H^{o}\left(\rho_{[1, T]}^{-}\right) \prod_{0 \leq i<j \leq T} H\left(\rho_{i}^{+} ; \rho_{j}^{-}\right) \\
& =\prod_{1 \leq i<j \leq 2 p} \frac{1}{1-x_{i} x_{j}} \prod_{i=1}^{2 p} H\left(\rho_{[1, T)}^{-} \cup \rho_{[0, T)}^{+} ; x_{i}\right) \cdot H^{o}\left(\rho_{[1, T)}^{-}\right) \prod_{0 \leq i<j \leq T-1} H\left(\rho_{i}^{+} ; \rho_{j}^{-}\right)
\end{aligned}
$$

Furthermore, for $k<l$, up to terms of high degree we have

$$
\begin{aligned}
& \frac{\left(x_{1} \cdots \hat{x}_{k} \cdots \hat{x}_{l} \cdots x_{2 p}\right)^{2 p}}{\prod_{1 \leq i<j \leq 2 p, i, j \neq k, l}\left(x_{i}-x_{j}\right)} \operatorname{Pf} N\left(\begin{array}{l}
1 \cdots \hat{k} \cdots \hat{l} \cdots 2 p \\
1 \cdots \hat{k} \cdots \hat{l} \cdots 2 p
\end{array}\right) \\
& =\prod_{1 \leq i<j \leq 2 p} \frac{1}{1-x_{i} x_{j}} \prod_{i=1}^{2 p} H\left(\rho_{[1, T)}^{-} \cup \rho_{[0, T)}^{+} ; x_{i}\right) \cdot H^{o}\left(\rho_{[1, T)}^{-}\right) \prod_{0 \leq i<j \leq T-1} H\left(\rho_{i}^{+} ; \rho_{j}^{-}\right) \\
& \times \frac{\prod_{i=1}^{2 p}\left(1-x_{i} x_{k}\right)\left(1-x_{i} x_{l}\right)}{\left(1-x_{k}^{2}\right)\left(1-x_{l}^{2}\right)\left(1-x_{k} x_{l}\right) H\left(\rho_{[1, T)}^{-} \cup \rho_{[0, T)}^{+} ; x_{k}, x_{l}\right)}
\end{aligned}
$$

and

$$
\begin{aligned}
\left(N^{-1}\right)_{k l}=(-1)^{k+l} \frac{\operatorname{Pf} N\left(\begin{array}{c}
1 \cdots \hat{k} \cdots \hat{l} \cdots 2 p \\
1 \ldots \hat{k} \cdots \hat{l} \cdots 2 p
\end{array}\right)}{\operatorname{Pf} N}=\frac{\left(x_{l}-x_{k}\right) x_{k} x_{l}}{\prod_{i \neq k}\left(1-x_{i} / x_{k}\right) \prod_{j \neq l}\left(1-x_{j} / x_{l}\right)} \\
\times \frac{\prod_{i=1}^{2 p}\left(1-x_{i} x_{k}\right)\left(1-x_{i} x_{l}\right)}{\left(1-x_{k}^{2}\right)\left(1-x_{l}^{2}\right)\left(1-x_{k} x_{l}\right) H\left(\rho_{[1, T)}^{-} \cup \rho_{[0, T)}^{+} ; x_{k}, x_{l}\right)} .
\end{aligned}
$$


Hence,

$$
\begin{aligned}
\left(\Xi N^{-1} \Xi^{t}\right)_{u v} & =\sum_{k, l=1}^{2 p} \frac{\left(x_{l}-x_{k}\right) x_{k}^{u+1} x_{l}^{v+1}}{\prod_{i \neq k}\left(1-x_{i} / x_{k}\right) \prod_{j \neq l}\left(1-x_{j} / x_{l}\right)} \\
& \times \frac{\prod_{i=1}^{2 p}\left(1-x_{i} x_{k}\right)\left(1-x_{i} x_{l}\right)}{\left(1-x_{k}^{2}\right)\left(1-x_{l}^{2}\right)\left(1-x_{k} x_{l}\right) H\left(\rho_{[1, T)}^{-} \cup \rho_{[0, T)}^{+} ; x_{k}, x_{l}\right)} \\
= & \frac{1}{(2 \pi i)^{2}} \oint \oint \frac{(w-z) H\left(\rho_{T}^{-} ; z^{-1}, w^{-1}\right) z^{u} w^{v}}{\left(1-z^{2}\right)\left(1-w^{2}\right)(1-z w) H\left(\rho_{[1, T)}^{-} \cup \rho_{[0, T)}^{+} ; z, w\right)} d z d w
\end{aligned}
$$

The integral is understood as the sum of residues at the points $z, w=x_{1}, \ldots, x_{2 p}$. Taking convolutions of this expression with $V_{i}$ 's, which are Toeplitz matrices with symbols $H\left(\rho_{i}^{-} ; z\right) H\left(\rho_{i}^{+} ; z^{-1}\right)$, and with $\epsilon$ which is also Toeplitz with symbol $\left(z^{-1}-\right.$ $z) H\left(\rho_{0}^{+} ; z\right) H\left(\rho_{0}^{+} ; z^{-1}\right)$, we obtain, in the notation of (1.7),

$$
\begin{aligned}
& \left(V_{[i, T)} \Xi N^{-1} \Xi^{t} V_{[j, T)}^{t}\right)_{u v} \\
= & \frac{1}{(2 \pi i)^{2}} \iint \frac{(w-z) z^{u} w^{v}}{\left(1-z^{2}\right)\left(1-w^{2}\right)(1-z w)} \frac{H\left(\rho_{[i, T]}^{-} ; z^{-1}\right) H\left(\rho_{[j, T]}^{-} ; w^{-1}\right)}{H\left(\rho_{[1, T]}^{-} \cup \rho_{[0, i)}^{+} ; z\right) H\left(\rho_{[1, T]}^{-} \cup \rho_{[0, j)}^{+} ; w\right)} d z d w .
\end{aligned}
$$

Inverting the variables of integration yields the expression for $K_{11}$.

Furthermore,

$$
\begin{aligned}
& \left(V_{[i, T)} \Xi N^{-1} \Xi^{t} V_{[1, T)}^{t} \epsilon V_{[1, j)}\right)_{u v} \\
& \quad=\frac{1}{(2 \pi i)^{2}} \iint \frac{(w-z) z^{u} w^{v}}{\left(1-z^{2}\right) w(1-z w)} \frac{H\left(\rho_{[i, T]}^{-} ; z^{-1}\right) H\left(\rho_{[1, T]}^{-} \cup \rho_{[0, j)}^{+} ; w^{-1}\right)}{H\left(\rho_{[1, T]}^{-} \cup \rho_{[0, i)}^{+} ; z\right) H\left(\rho_{[j, T]}^{-} ; w\right)} d z d w .
\end{aligned}
$$

Note that the residue of this integral at $w=z^{-1}$ equals $(i<j)$

$$
-\frac{1}{2 \pi i} \int H\left(\rho_{[i, j)}^{-} ; z^{-1}\right) H\left(\rho_{[i, j)}^{+} ; z\right) z^{u-v-1} d z=\left(-V_{[i, j)}\right)_{u v}
$$

which is the second term in the $(1,2)$-entry of (1.7). This proves the formula for $K_{21}$ and $K_{12}$.

Finally,

$$
\begin{aligned}
& \left(-V_{[1, i)}^{t} \in V_{[1, T)} \Xi N^{-1} \Xi^{t} V_{[1, T)}^{t} \in V_{[1, j)}\right)_{u v} \\
& =\frac{1}{(2 \pi i)^{2}} \iint \frac{(w-z) z^{u} w^{v}}{z w(1-z w)} \frac{H\left(\rho_{[1, T]}^{-} \cup \rho_{[0, i)}^{+} ; z^{-1}\right) H\left(\rho_{[1, T]}^{-} \cup \rho_{[0, j)}^{+} ; w^{-1}\right)}{H\left(\rho_{[i, T]}^{-} ; z\right) H\left(\rho_{[j, T]}^{-} ; w\right)} d z d w,
\end{aligned}
$$

and the residue of the integral at $w=z^{-1}$ gives

$$
\frac{1}{2 \pi i} \int\left(z-z^{-1}\right) H\left(\rho_{[1, j)}^{-} \cup \rho_{[0, i)}^{+} ; z^{-1}\right) H\left(\rho_{[1, i)}^{-} \cup \rho_{[0, j)}^{+} ; z\right) z^{u-v-1} d z=\left(V_{[1, i)}^{t} \epsilon V_{[1, j)}\right)_{u v}
$$

as is needed in the $(2,2)$-block of (1.7). 


\section{AbStract processes}

Given a conditional determinantal or Pfaffian $L$-ensemble, the associated collection of minors determines a point in $\mathbb{R}^{2^{n}}=\left(\mathbb{R}^{2}\right)^{\otimes n}$. For algebraic purposes, it is nicer to consider the complex analogue of this notion; we thus obtain the following definitions.

Definition 4.1. Let $n$ be a positive integer. A nonzero point $p \in\left(\mathbb{C}^{2}\right)^{\otimes n}$ is determinantal if there exists an integer $m \geq 0$ and an $(n+m) \times(n+m)$ matrix $K$ such that for $S \subset\{1,2, \ldots n\}$,

$$
p_{S}=\underset{S \cup\{n+1, \ldots, n+m\}}{\operatorname{det}}(K) .
$$

The point is Pfaffian if there exists a $2 \times 2$ matrix valued $(n+m) \times(n+m)$ skew-symmetric matrix $K$ such that

$$
p_{S}=\operatorname{Pf}_{S \cup\{n+1, \ldots, n+m\}}(K)
$$

for all $S \subset\{1,2, \ldots n\}$.

A point process whose correlation functions $\rho(S)$ are given by minors $p_{S}$ as above is called conditional determinantal (or conditional Pfaffian).

If we replace $K$ by the block matrix

$$
\left[\begin{array}{cc}
K & 0 \\
0 & s
\end{array}\right] \text {, respectively }\left[\begin{array}{ccc}
K & 0 \\
0 & {\left[\begin{array}{cc}
0 & s \\
-s & 0
\end{array}\right]}
\end{array}\right],
$$

for some nonzero scalar $s$, this simply multiplies $p_{S}$ by $s$, and thus the conditions depend only on the corresponding points in the projective space $\mathbb{P}^{2^{n}-1}(\mathbb{C})$.

Now, adding a multiple of one of the last $m$ rows/columns of $K$ to one of the first $n$ rows/columns of $K$ leaves $p$ unchanged, as does an arbitrary change of basis applied to the last $m$ rows/columns. It follows that we may choose $K$ of the form

$$
\left[\begin{array}{ccc}
A & B & 0 \\
C & 0 & 0 \\
0 & 0 & D
\end{array}\right]
$$

where $A$ is $n \times n$ and $D$ is diagonal and invertible. But this gives the same point, projectively, as

$$
\left[\begin{array}{ll}
A & B \\
C & 0
\end{array}\right]
$$

It follows that in the definition of determinantal or Pfaffian points, it suffices to consider $m=n+1$. In particular, the set of such points is an algebraic set, as the image of the space of matrices under a polynomial map.

Theorem 4.2. The set of determinantal (resp. Pfaffian) points in $\mathbb{P}^{n}-1(\mathbb{C})$ is invariant under the natural action of the group $\mathrm{GL}_{2}(\mathbb{C})^{n} \ltimes S_{n}$.

Here the $j$ th copy of $\mathrm{GL}_{2}(\mathbb{C})$ acts on $p$ by

$$
\begin{gathered}
\left(\left[\begin{array}{ll}
a & b \\
c & d
\end{array}\right]_{j} p\right)_{S}=a p_{S}+b p_{S \cup\{j\}} \\
\left(\left[\begin{array}{ll}
a & b \\
c & d
\end{array}\right]_{j} p\right)_{S \cup j}=c p_{S}+d p_{S \cup\{j\}},
\end{gathered}
$$


and $S_{n}$ acts in the obvious way (permuting the tensor factors).

Proof. We consider the determinantal case; the Pfaffian case is analogous.

The invariance under $S_{n}$ is immediate, and thus invariance under the full group will follow from invariance under the first copy of $\mathrm{GL}_{2}(\mathbb{C})$.

Suppose $p$ is determinantal, with kernel

$$
K=\left[\begin{array}{ccc}
a & \vec{b} & \vec{c} \\
\vec{d} & E & F \\
\vec{g} & H & M
\end{array}\right] .
$$

Multiplying the first row or first column by $\alpha$ replaces $p$ by

$$
\left[\begin{array}{ll}
1 & 0 \\
0 & \alpha
\end{array}\right]_{1} p
$$

and thus the latter is determinantal. Similarly, replacing $a$ by $a+a_{0}$ takes $p$ to

$$
\left[\begin{array}{cc}
1 & 0 \\
a_{0} & 1
\end{array}\right]_{1} p
$$

We thus have invariance under a Borel subgroup of $\mathrm{GL}_{2}(\mathbb{C})$; it will thus suffice to consider the corresponding Weyl group. In other words, we need to show invariance under

$$
\left[\begin{array}{ll}
0 & 1 \\
1 & 0
\end{array}\right]_{1}
$$

in particular, that determinantal/Pfaffian processes are closed under taking symmetric differences. ${ }^{4}$ In fact, $\left(\left[\begin{array}{ll}0 & 1 \\ 1 & 0\end{array}\right]_{1} p\right)$ can be obtained from the $(n+m+1) \times$ $(n+m+1)$ kernel

$$
K^{\prime}=\left[\begin{array}{cccc}
0 & 0 & 0 & 1 \\
0 & E & F & \vec{d} \\
0 & H & M & \vec{g} \\
-1 & \vec{b} & \vec{c} & a
\end{array}\right]
$$

The invariance claim follows.

Remark 4.3. Note in particular that for any probability distribution $p$,

$$
\left(\left[\begin{array}{ll}
1 & 1 \\
0 & 1
\end{array}\right]^{\otimes n} p\right)_{S}=\sum_{T \supset S} p_{S},
$$

which should be viewed as the correlation function of $p$. We thus arrive at an a priori nonobvious conclusion that every conditional determinantal (Pfaffian) process is a conditional determinantal (Pfaffian) $L$-ensemble and vice versa. (Note that the converse statement also follows from Proposition 1.2.)

Let $D_{n}$ be the topological closure of the set of determinantal points, and let $P_{n}$ be the closure of the set of Pfaffian points; of course both of these are projective

\footnotetext{
${ }^{4}$ The observation that the set of determinantal processes is invariant under taking symmetric differences is due to Kerov, cf. [BOO, A.3].
} 
varieties. Now, the generic point of either set satisfies $p_{\emptyset} \neq 0$, and thus can be obtained from an $n \times n$ kernel. Thus naïvely, we should have the dimensions

$$
\operatorname{dim}\left(D_{n}\right) \sim n^{2}-n+1, \quad \operatorname{dim}\left(P_{n}\right) \sim n(2 n-1)-3 n,
$$

in each case the difference of the dimension of the space of kernels and the dimension of the set of "equivalent" kernels. (For $D_{n}$, this entails conjugation by diagonal matrices, while for $P_{n}$, it entails the natural action of $\mathrm{SL}_{2}(\mathbb{C})^{n}$ on the kernel.) Of course, if there exist inequivalent kernels for the same point, or if the generic kernel has an automorphism, these formulas fail, but this happens only for small $n$. We in fact have the following.

Theorem 4.4. For all $n, \operatorname{dim}\left(D_{n}\right)=n^{2}-n+1$; in particular, for $n \leq 3, D_{n}=$ $\mathbb{P}^{2^{n}-1}(\mathbb{C})$. Similarly, for $n \geq 5$, $\operatorname{dim}\left(P_{n}\right)=2 n(n-2)$, while for $n \leq 4, P_{n}=$ $\mathbb{P}^{2^{n}-1}(\mathbb{C})$.

Proof. For $D_{n}$, the generic $n \times n$ kernel has a canonical form (in which the offdiagonal entries of the first row are all 1 ), from which we readily determine that it has no automorphisms, and is uniquely determined by the associated point (in fact by the coordinates of that point on sets of size $\leq 2$ ). Thus the naïve dimension count is in fact accurate.

For $P_{n}$, both properties fail for small $n$. For $n=1$, every kernel is invariant under $\mathrm{SL}_{2}(\mathbb{C})$, while for $n=2$, the generic kernel can be taken to the form

$$
\left[\begin{array}{cccc}
0 & a & 0 & -b \\
-a & 0 & b & 0 \\
0 & -b & 0 & -c \\
b & 0 & c & 0
\end{array}\right],
$$

invariant under the diagonal subgroup of $\mathrm{SL}_{2}(\mathbb{C})^{2}$. For $n=3$, the generic kernel still has a 1-dimensional automorphism group; finally for $n \geq 4$, the generic kernel has no automorphisms. Since for $n \leq 3, P_{n} \supset D_{n}=\mathbb{P}^{2^{n}-1}(\mathbb{C})$, we have $\operatorname{dim}\left(P_{n}\right)=2^{n}-1$ for $n \leq 3$, and thus the generic automorphism group is the only correction to the dimension formula; in particular, the generic point in $P_{1}, P_{2}, P_{3}$ determines a unique kernel up to equivalence.

For $n=4$, the above dimension count is too high; it gives 16 out of a possible 15 , suggesting that the generic point determines a one-parameter family of equivalence classes of kernels. By direct computation with a random Pfaffian point, one can show the existence of a point with such a family, showing that $\operatorname{dim}\left(P_{4}\right) \geq 15$ and thus $P_{4}=\mathbb{P}^{2^{n}-1}(\mathbb{C})$.

Similarly, for $n=5$, it suffices to find a (random) point having a unique kernel up to equivalence; the lack of automorphisms gives rise to a canonical form, showing that this uniqueness extends to all larger $n$.

The first nontrivial instances are thus $D_{4}$ and $P_{5}$. The structure of $D_{4}$ can be deduced from the following fact.

Proposition 4.5. Let $p \in \mathbb{P}^{2^{4}-1}(\mathbb{C})$ be a point such that $p_{S}=0$ unless $|S|=2$. Then $p$ is determinantal.

Proof. Equivalently, we may assume that $p$ is supported on the six sets

$$
\emptyset,\{1,2,3,4\},\{1,3\},\{1,4\},\{2,3\},\{2,4\} ;
$$


and by symmetry and rescaling assume that $p_{\emptyset}=1$. But then the kernel

$$
\left[\begin{array}{cccc}
0 & 0 & 1 & -p_{\{1,4\}} \\
0 & 0 & \frac{-p_{\{2,3\}}}{x} & 1 \\
-p_{\{1,3\}} & x & 0 & 0 \\
1 & -p_{\{2,4\}} & 0 & 0
\end{array}\right]
$$

works, for a suitable choice of $x$.

Theorem 4.6. A point in $\mathbb{P}^{2^{4}-1}(\mathbb{C})$ is determinantal if and only if it is in the $\mathrm{GL}_{2}(\mathbb{C})^{\otimes 4}$-orbit of a point supported on sets of size 2. Equivalently, $D_{4}$ is the codimension 2 variety $\nabla_{\text {node }}(\emptyset)$, in the notation of [WZ], where we have identified $\mathbb{C}^{2^{4}}$ with the space of multilinear polynomials on $\left(\mathbb{P}^{1}\right)^{4}$; in other words, $D_{4}$ is the variety of multilinear polynomials with two critical points in general position.

Proof. Given a multilinear polynomial with two critical points in general position, we may act by $\mathrm{GL}_{2}(\mathbb{C})^{\otimes 4}$ to put the critical points at $(0,0,0,0),(\infty, \infty, \infty, \infty)$; but then the corresponding point in $\mathbb{P}^{15}(\mathbb{C})$ is determinantal by the proposition. The remaining claims follow by comparing dimensions.

Note that although this gives a fairly simple direct characterization of $D_{4}$, the variety itself is fairly complicated. In fact, one can show that the variety has degree 28 , with ideal generated by a whopping 718 degree 12 polynomials.

For $P_{5}$, the situation is even worse; although dimension considerations show that $P_{5}$ is a hypersurface, and thus cut out by a single $\mathrm{GL}_{2}$-invariant polynomial, experimentation over finite fields suggests that this polynomial has degree 1146 . We have also been unable to find any sort of natural direct characterization of $P_{5}$.

\section{REFERENCES}

[B] A. Borodin, Biorthogonal ensembles, Nuclear Physics B 536 (1999), 704-732, math.CA/ 9804027.

[BOO] A. Borodin, A. Okounkov, G. Olshanski, Asymptotics of Plancherel measures for symmetric groups, J. Amer. Math. Soc. 13 (2000), 491-515, math/9905032.

[DVJ] D. J. Daley, D. Vere-Jones, An introduction to the theory of point processes, Springer series in statistics, Springer, 1988.

[EM] B. Eynard and M. L. Mehta, Matrices coupled in a chain. I. Eigenvalue correlations, J. Phys. A: Math. Gen. 31 (1998), 4449-4456, cond-mat/9710230.

[FNH] P. J. Forrester, T. Nagao, G. Honner, Correlations for the orthogonal-unitary and symplectic-unitary transitions at the hard and soft edges, Nucl. Phys. B 553 (1999), no. 3, 601-643, cond-mat/9811142.

[J1] K. Johansson, Random growth and Random matrices, European Congress of Mathematics, Barcelona, vol. I, Birkhäuser, 2001.

[J2] , Discrete polynuclear growth and determinantal processes, Comm. Math. Phys. 242 (2003), 277-329; math.PR/0206208.

[J3] - The Arctic circle boundary and the Airy process, math.PR/0306216.

[J4] Non-intersecting, simple, symmetric random walks and the extended Hahn kernel, math.PR/0409013.

[Ma] O. Macchi, The coincidence approach to stochastic point processes, Adv. Appl. Prob. 7 (1975), 83-122.

[M] I. G. Macdonald, Symmetric functions and Hall polynomials, 2nd edition, Oxford University Press, 1995.

[Mat1] S. Matsumoto, Correlation functions of the shifted Schur measure, math.C0/0312373,

[Mat2] _ Alpha-Pfaffian, Pfaffian point process and shifted Schur measure, nath.C0/0411277.

[NF1] T. Nagao and P. J. Forrester, Multilevel Dynamical Correlation Function for Dyson's Brownian Motion Model of Random Matrices, Phys Lett. A 247 (1998), 42-46. 
EYNARD-MEHTA THEOREM, SCHUR PROCESS, AND THEIR PFAFFIAN ANALOGS21

[NF2] - Quaternion determinant expressions for multilevel dynamical correlation functions of parametric random matrices, Nucl. Phys. B 563 (1999), no. 3, 547-572.

[OR] A. Okounkov and N. Reshetikhin, Correlation functions of Schur process with applications to local geometry of a random 3-dimensional Young diagram, J. Amer. Math. Soc. 16 (2003), 581-603, math.C0/0107056.

[R] E. Rains, Correlation functions for symmetrized increasing subsequences, math.C0/0006097

[SI] T. Sasamoto and T. Imamura, Fluctuations of a one-dimensional polynuclear growth model in a half space, J. Stat. Phys. 115 (2004), no. 3-4, 749-803, cond-math/0307011.

[TW1] C. A. Tracy and H. Widom, Correlation functions, cluster functions and spacing distributions for random matrices, J. Stat. Phys. 92 (1998), 809-835, solv-int/9804004.

[TW2] - Differential equations for Dyson processes, Comm. Math. Phys. 252 (2004), no. 1$3,7-41$, math.PR/0309082.

[TW3] _ A limit theorem for shifted Schur measures, Duke Math. J 123 (2004), no. 1, 171-208, math.PR/0210255.

[WZ] J. Weyman and A. Zelevinsky, Singularities of hyperdeterminants, Ann. Inst. Fourier (Grenoble) 46 (1996), no. 3, 591-644. 